Article

\title{
Pre-Processing of Energy Demand Disaggregation Based Data Mining Techniques for Household Load Demand Forecasting
}

\author{
Ahmed F. Ebrahim and Osama A. Mohammed * (D) \\ Energy Systems Research Laboratory, Department of Electrical \& Computer Engineering, Florida International \\ University, Miami, FL 33174, USA; aebra003@fiu.edu \\ * Correspondence: mohammed@fiu.edu; Tel.: +1-305-348-3040
}

Received: 3 June 2018; Accepted: 4 July 2018; Published: 8 July 2018

\begin{abstract}
Demand side management has a vital role in supporting the demand response in smart grid infrastructure, in the decision-making of energy management, in household applications is significantly affected by the load-forecasting accuracy. This paper introduces an innovative methodology to enhance household demand forecasting based on energy disaggregation for Short Term Load Forecasting. This approach is constructed from Feed-Forward Artificial Neural Network forecaster and a pre-processing stage of energy disaggregation. This disaggregation technique extracts the individual appliances' load demand profile from the aggregated household load demand to increase the training data window for the proposed forecaster. These proposed algorithms include two benchmark disaggregation algorithms; Factorial Hidden Markov Model (FHMM), Combinatorial Optimization in addition to three adopted Deep Neural Network; long short- term memory (LSTM), Denoising Autoencoder, and a network which regress start time, end time, and average power. The proposed load forecasting approach outperformed the currently available state-of-the-art techniques; namely root mean square error (RMSE), normalized root mean square error (NRMSE), and mean absolute error (MAE).
\end{abstract}

Keywords: household load forecasting; non-intrusive load-monitoring (NILM); feed-forward artificial neural network (FFANN); deep learning (DL); data mining (DM)

\section{Introduction}

The innovations at different power system infrastructures' levels facilitate the integration of new smart grid ideas. However, new architectures of smart grid add an extra burden on the grid regarding complexity and uncertainty. As a result of the increased penetration of renewable energy, Electric Vehicles (EVs), and time-varying loads in the distribution system, the grid will be vulnerable to unusual, challenging experiences for utility-customer interactions. Household loads represent a significant percentage of electrical energy consumption. The households' demand-side response (DSR) enable active participation of these loads in the grid enhancing power system stability.

Consequently, the forecasting of household energy consumption is crucial for household DSR programs. Precise short-term load forecasting (STLF) has a significant effect on the accuracy of the household DSR. However, STLF is challenging at this level of the grid due to uncertainty and volatility in load consumption originating from customer behavior, which is too stochastic to predict.

Common techniques, such as exponential smoothing, autoregressive integrated moving average (ARIMA) based time-series analysis [1], support vector machine (SVM) [2] and feed-forward artificial neural networks (FFANN) based machine learning have been used in the literature to achieve good STLF forecasting [3]. 
An adopted ARIMA model for a day ahead load forecasting was presented in [4], in which the forecasting technique is based on grouping the targeted day with similar meteorological days in historical data. A radial basis function (RBF) neural network was used for STLF in [5]. An adaptive neural fuzzy inference system (ANFIS) was combined with RBF neural network to adjust the forecasting by taking into consideration real-time electricity prices [6]. A neural network based predictor for STLF was presented in [7]. The latter uses the load values of the current and previous time steps as inputs to predict the load value at the subsequent time step. A forecaster for the total load of the Australian national energy market was based on an ensemble of extreme learning machines (ELMs) is suggested in [8]. A committed input choice structure to work with the hybrid prediction framework using the Bayesian neural network and wavelet transformation was introduced in [9].

Based on the current state-of-the-art, the procedures used for load forecasting can be classified into three categories. The first is to evade the uncertainty by clustering/classification techniques which gather comparable customers, days or weather in the hope of decreasing the variance of uncertainty within each cluster [10]. However, the accuracy of this technique is heavily dependent on the amount of available data. The second category is using the aggregated smart metering data to cancel out the uncertainty. Therefore, the aggregated load exhibits typically regular patterns and more accessible to predict. However, the accuracy of this technique is heavily dependent on an aggregated level of data. The third category is separating the regular pattern from the other component of load profile such as uncertainty and noise by pre-processing techniques, mostly spectral analysis such as empirical mode decomposition (EMD) [11], Fourier transforms [12], and wavelet analysis [13]. These techniques are unsuitable for the household load forecasting due to the high uncertainty proportion of the load pattern.

All these previous techniques are appropriate for higher grid levels such as community or system levels. However, there are few works done in the literature on STLF at the household level. In [14,15] a time series forecasting approach was presented. However, it used a daily median absolute error (DMAE) which is not the commonly used performance metric. Therefore, it is improper for use as a benchmark for preliminary assessments. Mean absolute percentage error (MAPE) is the standard metric used to assess the forecaster performance. Recently, deep artificial neural network (DANN) used in household load forecasting [14]. The DANN technique used is a factored conditionally restricted Boltzmann machine. The latter improves performance rather than support vector machine and artificial neural network. Another deep neural network (DNN) approach called long short-term memory (LSTM) was used in [16]. Although the high expectation in forecasting community, the current state of the art indicates that deep learning is more prone to over-fitting compared with artificial neural networks [7]. This issue is expected due to the existence of more parameters and relatively fewer data. For that reason, another work based on a pooling-based deep recurrent neural network (PDRNN) was proposed in [17] to tackle the overfitting issue. However, the procedure was an attempt to tackle the over-fitting issue by increasing the training data window dimension which is the historical data of the neighbors to the household system under study. The main drawback here is that the PDRNN method pools the data of the neighboring smart meters to enlarge the training widow dataset which most probably is unavailable for privacy concerns.

In this paper, an innovative methodology for STLF of household load demand is developed and employed. This approach is constructed from Feed-Forward Artificial Neural Network (FFANN), and a pre-processing Stage of Energy Disaggregation (SOED) based Data Mining Algorithms (DMA). This SOED extracts the individual appliances' load demand profile from the aggregated household load demand to increase the training data window for the FFANN forecaster. These DMA include two bench-mark disaggregation algorithms (Factorial Hidden Markov Model (FHMM), Combinatorial Optimization) and three adopted Deep Neural Network (Long Short-Term Memory (LSTM), Denoising Autoencoder (DAE), and a network which regress start time, end time, and average power (RECTANGLES)). The proposed load forecasting approach outperforms the current state of the art techniques such as ARIMA, SVM, and FFANN regarding RMSE, NRMSE, and MAE. The main 
contributions of this paper are summarized in the following: (1) The improved forecasting architecture that combines the neural network and energy disaggregation to improve the forecasting for challenging load patterns such as household and small microgrid with high uncertainty and a small amount of historical data; (2) the second contribution of the paper is the detailed analysis and comparison of different disaggregation and forecasting algorithm; (3) the proposed algorithm target small microgrids and residential loads level. Improvement of the load forecasting for such loads is necessary for better energy management and demand-side management. The real-time pricing for such loads usually changes in hourly bases [18,19]. This paper is organized as follows: Section 2 describes the energy disaggregation system and its implementation. In Section 3, a description of the proposed Short-Term Load Forecasting approach is illustrated. In Section 4, simulation results are presented and investigated to validate the proposed forecaster. Finally, in Section 5, some conclusions are deduced from the developments in this paper.

\section{Energy Disaggregation}

Energy disaggregation (ED) is a computational approach for predicting the individual appliances power demand from a single meter which measures the aggregated power demand. George Hart starts this research in the mid-1980s [20,21]. His earliest research defined a signature taxonomy of feature. Nevertheless, his concentration was on extracting only transitions between steady-states. Consequent Hart's clues, several ED procedures prepared for low-frequency data (1 Hz or slower) only to extract a minor number of features. There are numerous instances in the literature of manual feature extractors regarding the high-frequency sampling at $\mathrm{kHz}$ or even $\mathrm{MHz}$. [22,23]. Hand-engineer feature extractors for instance Difference of Gaussians (DoG) and scale-invariant feature transform (SIFT) was the leading method to mine features for image classification before 2012 [24]. However, in 2012, through the competition of ImageNet Large Scale Visual Recognition, several procedures achieved exceptional performance and did not use hand-engineered feature detectors. As an alternative, they used some disaggregation algorithms which automatically learned to extract a hierarchy of features from the raw image. In this paper, we will use five data mining algorithms for ED called CO, FHMM, DAE, LSTM, and RECTANGLES to extract the power demand profile for individual appliances from the main aggregated household power demand. Figure 1 shows the block diagram of the whole proposed system. The full illustration of these algorithms presented later in this section. To use ED to enhance household forecasting performance, the energy consumption of each household must be available. Therefore, the dataset from the UK-DALE was used [25]. Which is one of the first publicly available datasets collected essentially to support research on ED. It has a record of five houses. In our work, we will focus the study on only two houses. One of his recorded data was available during the training of the ED algorithm. However, the other was not seen during the training stage of the ED algorithm. Those five ED algorithms were implemented based on a toolkit called NILMTK [26]. The code is written in Python which offers a massive set of libraries supporting both machine learning and ED algorithms.

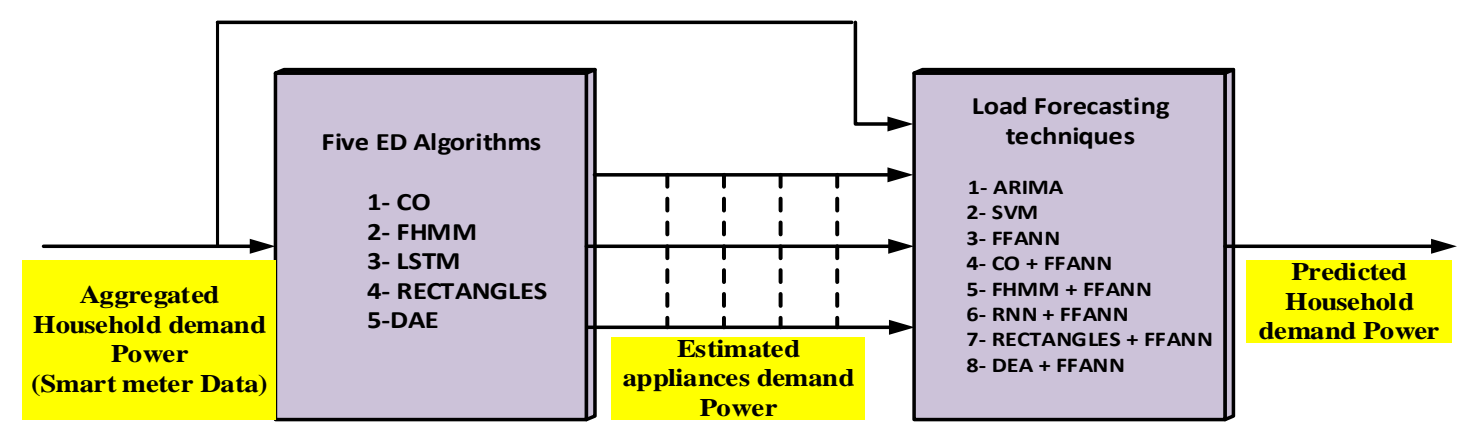

Figure 1. Block diagram for the proposed household load forecasting approach. 


\subsection{Data Mining Disaggregation Algorithms}

Five data mining disaggregation algorithms were used in this work. Two benchmark disaggregation algorithms; Factorial Hidden Markov Model (FHMM) and Combinatorial Optimization were utilized. Moreover, three adapted deep neural network architectures have been used for ED; (1) an exceptional form of a recurrent neural network (RNN) called long short-term memory (LSTM);

(2) a network that produces rectangles for the estimated demand by regression of the start time, end time and average power demand (nicknamed by RECTANGLES); and (3) denoising autoencoder (DAE). The full illustration of these algorithms is presented later in this section.

\subsubsection{Combinatorial Optimization}

Optimization methods necessitate the presence of appliance signature libraries with all possible groupings of power demands of the appliances it desires to disaggregate. If we include the gatherings of all the connected appliances in a house, then this optimization approach is called brute-force. However, due to memory limitations as stated in [27]. Brute-force methods are difficult to be applied to an embedded system. Therefore, the load identification requires the definition of an objective function and its minimization. Considering the aggregate data $\bar{x}$ and an appliance set $=\left[x_{1}, \ldots \ldots \ldots, x_{N}\right]$, the problem is formulated as [27].

$$
\min _{1 \leq n \leq N}\left\|\bar{x}-\sum_{n=1}^{N} x_{n}\right\|
$$

The Combinatorial Optimization is the most critical algorithm in this domain which minimizes the difference between the sum of the measure aggregate power and predicted appliance power [28]. This technique was used by Hart in [29]. The computational complexity is $\left(K^{N} T\right)$, where $K$ is the number of appliance states, $N$ the number of appliances and $T$ the number of times slices used in the implementation. Consequently, the optimization approaches address mainly disaggregation for the most power-hungry devices. Reference [30] discusses the two commonly cited disadvantages of this approach which are the decreasing of accuracy with the number of appliances and level of noise. Figure 2 shows the output result of the $\mathrm{CO}$ energy disaggregation algorithm. The figure divided into two columns. The left hand side (LHS) column has the analysis for the home whose data was available during the training of the disaggregation algorithm. The right hand side (RHS) column has the analysis for the home whose data was not available during the training of the disaggregation algorithm. The figure have six rows described as follows: (a) Aggregated power consumption for the home; (b) comparison between the estimated and Ground truth power demand for the dishwasher; (c) comparison between the estimated and Ground truth power demand for the Fridge; (d) comparison between the estimated and Ground truth power demand for the Kettle; (e) comparison between the estimated and Ground truth power demand for the Microwave; (f) comparison between the estimated and ground truth power demand for the washing machine.

\subsubsection{Factorial Hidden Markov Model}

FHMM belong to the group of Temporal Graphical Models which is a class of probabilistic models. Such models have been applied previously to many real-world problems such as speech recognition. The most direct demonstration of sequence data is through the use of a Markov chain which is a sequence of discrete variables. The state transitions of devices are controlled by the hidden Markov model (HMMs) which is a statistical tool. Each variable is defined by its real power consumption in addition to other useful information such as duration of the on and off periods and time of use during the day/week. Thereby, at an instant of time $t$ of a period $T, t \in T$, the aggregate consumption is $\bar{x}(t)$ and needs to be broken down to the number of appliances $z_{t}^{n}$, where $t \in T$ and $n \in N$ with $N$ the number of appliances. The value of each device $z_{t}^{n}$ at any time corresponds to one of the $K$ states of the trained model of the appliances [27]. The mathematical representation of the a HMM represented by Equation (2) through Equation (6) [27]. The behavior of a HMM can be completely defined and 
inferred by three parameters. First, the probability of each state of the hidden variable at the time $t$ can be represented by the vector $\pi$ such that

$$
\pi_{K}=\rho\left(z_{t}=k\right)
$$
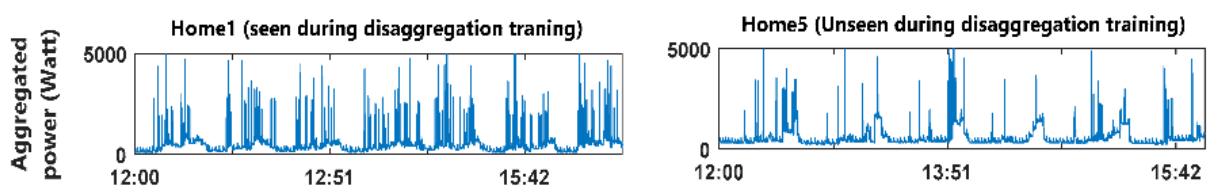

(a)
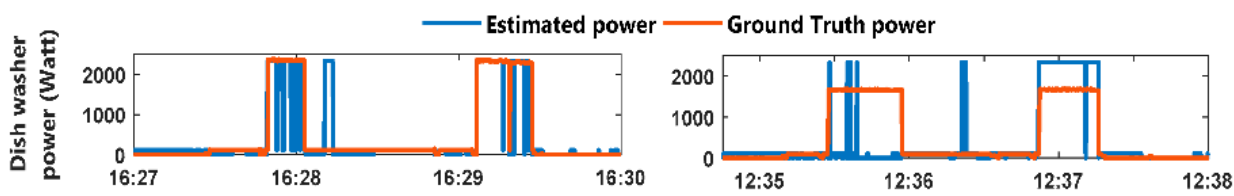

(b)
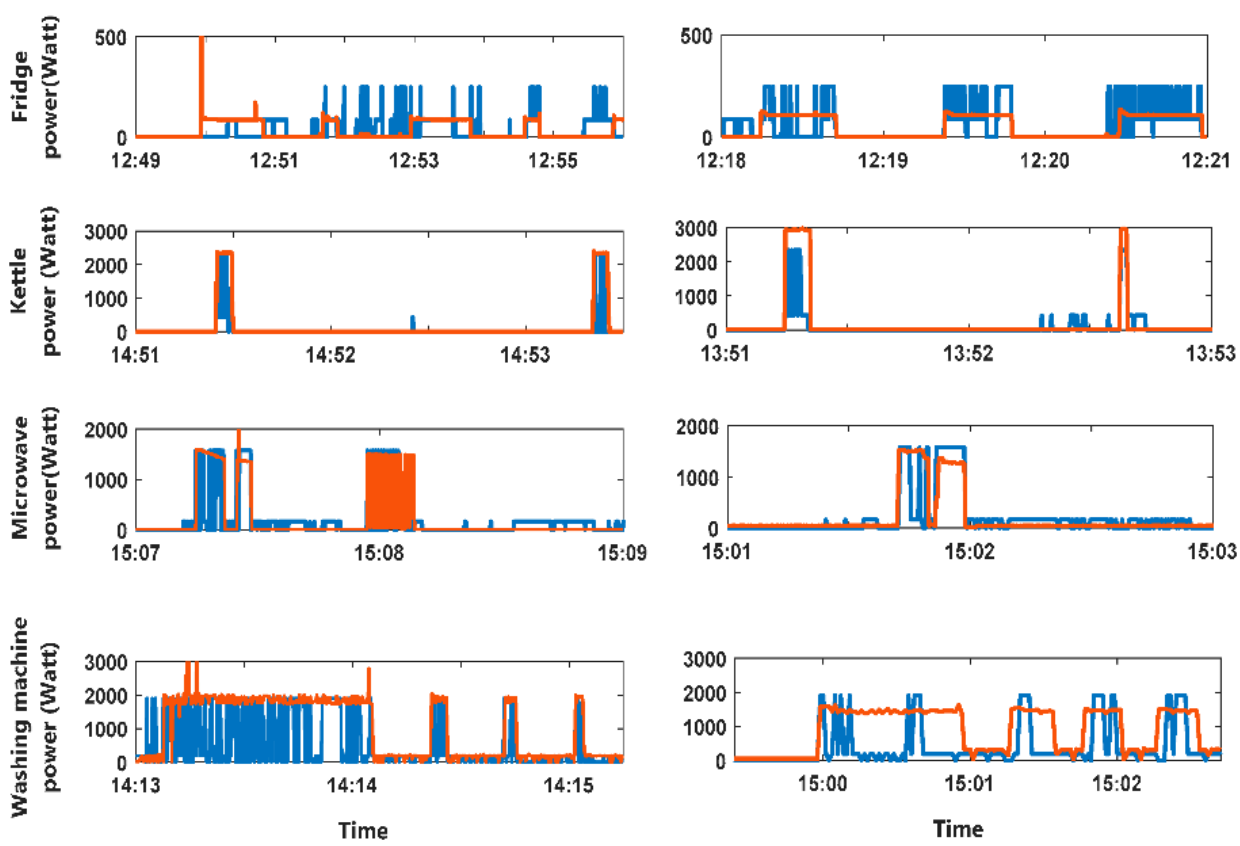

Figure 2. Shows the energy disaggregation algorithm output by using CO technique. (a) Aggregated power consumption for the home; (b) dishwasher estimated and Ground truth power demand; (c) fridge estimated and Ground truth power demand; (d) kettle estimated and Ground truth power demand; (e) microwave estimated and Ground truth power demand; (f) washing machine estimated and Ground truth power demand.

Second, the transition probabilities from state $i$ at $t$ to state $j$ at $t+1$ can be represented by the matrix $A$ such that,

$$
A_{i, j}=\rho\left(z_{t+1}=j \mid z_{t}=i\right)
$$

Third, the emission probabilities for $x$ are described by a statistical function with parameter $\varnothing$ which is commonly assumed to be Gaussian distributed such that,

$$
x_{t} \mid z_{t}, \varnothing \sim \mathcal{N}\left(\mu_{z_{t}}, \tau_{z_{t}}\right)
$$


where $\varnothing=\{\mu, \tau\}$, and $\mu_{z_{t}}, \tau_{z_{t}}$ are the mean and precision of a state's Gaussian distribution. Finally, Equations (2)-(4) can be used to compute the joint likelihood of a HMM:

$$
\rho(x, z \mid \theta)=\rho\left(z_{t} \mid \pi\right) \prod_{t=2}^{T} \rho\left(z_{t+1} \mid z_{t}, A\right) \prod_{t=1}^{T} \rho\left(x_{t} \mid z_{t}, \varnothing\right)
$$

where the set of all model parameters which must be found for each appliance during the training phase is represented by $\theta=\pi, A, \varnothing$. Therefore, when applying an HMM for Energy Disaggregation, it is needed to tune the $\theta$ parameters for each appliance during the training phase and afterwards, given a sequence of the power signal $\bar{x}$ to find the optimal sequence of discrete states $z$. Their ability to handle daily operation consumption and the information about state transition of devices makes them a suitable solution for the problem. The complexity of the disaggregation using HMMs is $O\left(K^{2} T\right)$, where $K$ is the number of states of all the appliances and $T$ is the number of the time slices, i.e., how many times the algorithm is required to be applied [27]. As it is shown the complexity is exponential with regard to the number of appliances while re-training is needed when a new group of appliances is added [31].

The HMMs were used for appliance load recognition, and it was also shown that they are useful in the field of ED [32]. Finally, HMMs is used to disaggregate an energy signal using generalized appliance model, and as a result, it was possible to extract consumption of individual devices without any manual labeling [25]. Nevertheless, the author uses low-frequency smart meter data because of lack of high-frequency data and smart metering infrastructure supporting such high rates. Although the HMM is a powerful technique, the method for the inference of hidden states is often affected by local minima [33]. To overcome this limitation, variants of HMMs are used such as the Factorial HMM (FHMM). The concept is that the output is an additive function of all the hidden states. In the model, each observation is dependent upon multiple unknown variables [34]. Likewise, the joint likelihood of an FHMM as stated in [35] is computed by,

$$
\rho\left(x^{(1: N)}, z \mid \theta\right)=\prod_{n=1}^{N} \rho\left(z_{t}^{(n)} \mid \pi\right) \prod_{t=2}^{T} \prod_{n=1}^{N} \rho\left(z_{t+1}^{(n)} \mid z_{t}^{(n)}, A\right) \prod_{t=1}^{T} \rho\left(x_{t} \mid z_{t}^{(1: N)}, \varnothing\right)
$$

where 1:N symbolizes a sequence of appliances $1, \ldots, N$. However, the computational complexity of both learning and disaggregating is greater for FHMMs compared to HMMs. This is due to the conditional dependence of the Markov chains.

Figure 3 shows the output result of the FHMM energy disaggregation algorithm. The figure divided into two columns. The LHS column has the analysis for the home whose data was available during the training of the disaggregation algorithm. The right-hand side (RHS column have the analysis for the home whose data was not available during the training of the disaggregation algorithm. The figure has six rows described as follows: (a) Aggregated power consumption for the home; (b) comparison between the estimated and Ground truth power demand for the dishwasher; (c) comparison between the estimated and ground truth power demand for the fridge; (d) comparison between the estimated and ground truth power demand for the kettle; (e) comparison between the estimated and Ground truth power demand for the microwave; (f) comparison between the estimated and Ground truth power demand for the washing machine. 

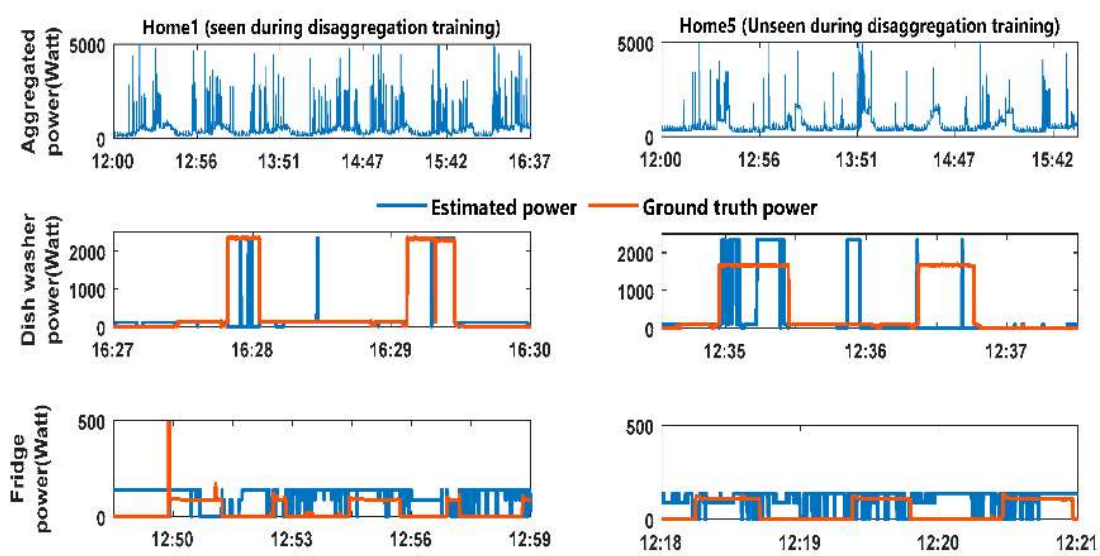

(c)
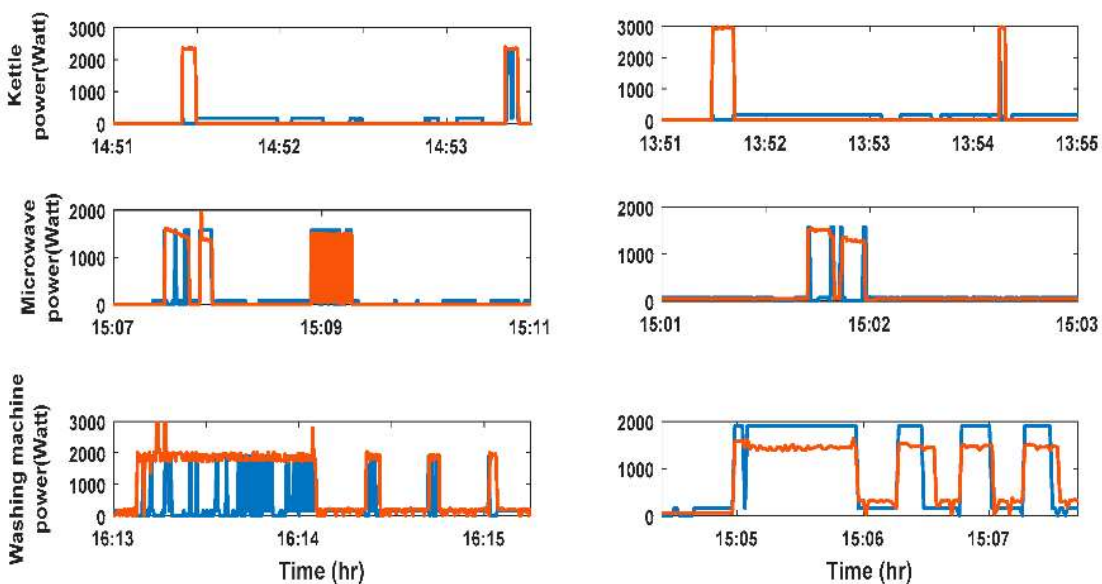

Figure 3. Shows the energy disaggregation algorithm output by using Factorial Hidden Markov Model (FHMM) technique. (a) Aggregated power consumption for the home; (b) dishwasher estimated and Ground truth power demand; (c) fridge estimated and Ground truth power demand; (d) kettle estimated and Ground truth power demand; (e) microwave estimated and Ground truth power demand; (f) washing machine estimated and Ground truth power demand.

\subsubsection{Denoising Autoencoder}

It is an autoencoder which attempts to reconstruct a clean target from a noisy input. DAEs are typically trained by an artificially corrupting signal before it goes into the net's input, where the net's target is the clean signal. In ED, we consider the corruption as being the power demand from the other appliances. So we do not add noise artificially. Instead, we use the aggregate power demand as the (noisy) input to the net and ask the net to reconstruct the clean power demand of the target appliance. Figure 4 shows the output result of the DAE energy disaggregation algorithm. The figure divided into two columns. The LHS column has the analysis for the home whose data was available during the training of the disaggregation algorithm. The right-hand side (RHS column have the analysis for the home whose data was not available during the training of the disaggregation algorithm. The figure has six rows described as follows: (a) Aggregated power consumption for the home; (b) comparison between the estimated and ground truth power demand for the dishwasher; (c) comparison between the estimated and ground truth power demand for the fridge; (d) comparison between the estimated and ground truth power demand for the kettle; (e) comparison between the estimated and ground truth power demand for the microwave; (f) comparison between the estimated and ground truth power demand for the washing machine. 

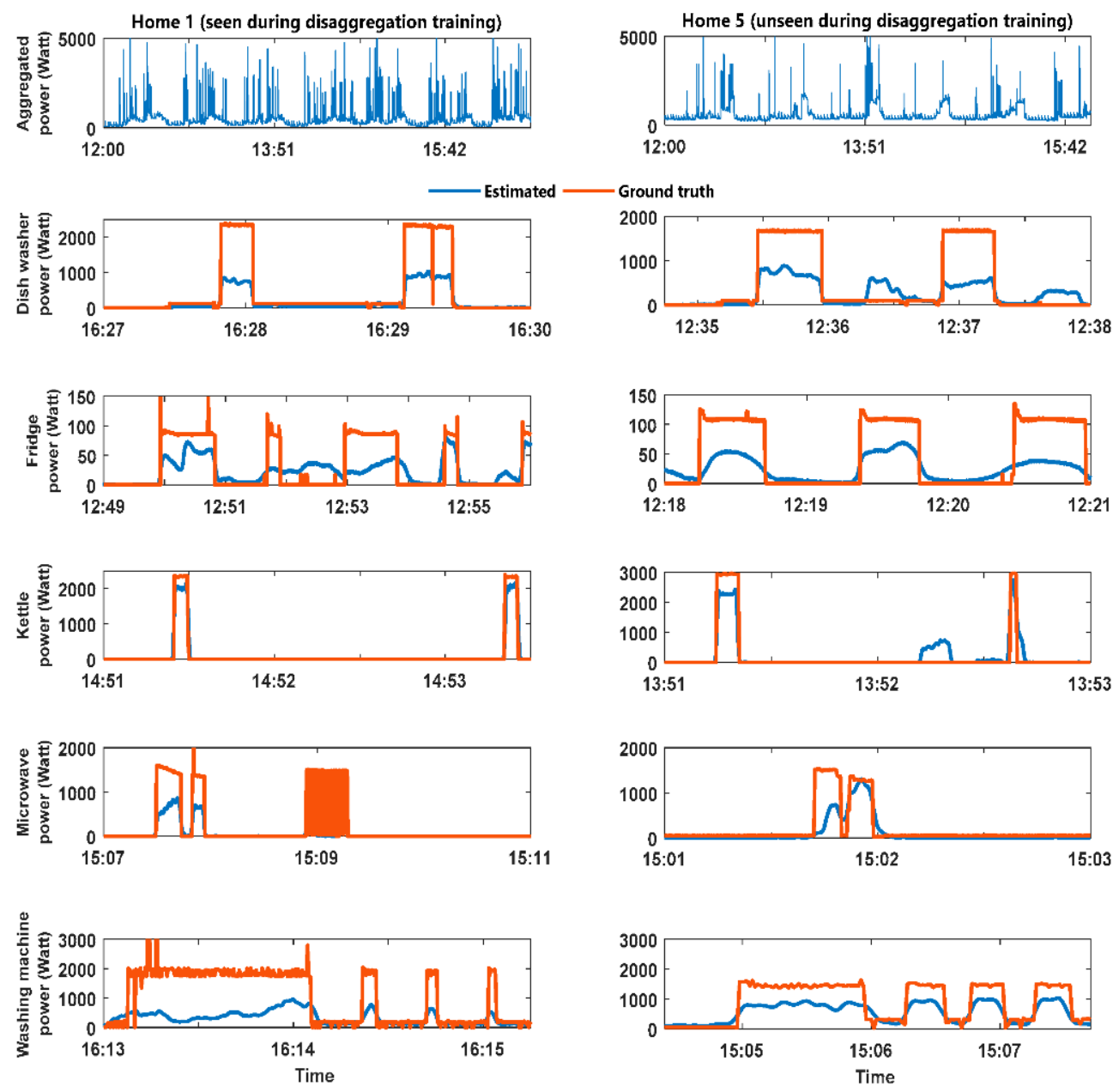

Figure 4. Shows the energy disaggregation algorithm output by using Denoising Autoencoder (DAE) technique. (a) Aggregated power consumption for the home; (b) dishwasher estimated and Ground truth power demand; (c) fridge estimated and Ground truth power demand; (d) kettle estimated and Ground truth power demand; (e) microwave estimated and Ground truth power demand; (f) washing machine estimated and Ground truth power demand.

\subsubsection{Regress Start Time, End Time, and Average Power (RECTANGLES)}

This algorithm draws a rectangle around each appliance activation in the aggregate data where the left side of the rectangle is the start time, the right side is the end time, and the height is the average power demand of the appliance between the start and end times.

Figure 5 shows the output result of the DAE energy disaggregation algorithm. The figure divided into two columns. The LHS column has the analysis for the home that its data was available during the training of the disaggregation algorithm. The right-hand side (RHS column have the analysis for the home whose data was not available during the training of the disaggregation algorithm. The figure has six rows described as follows: (a) Aggregated power consumption for the home; (b) comparison between the estimated and ground truth power demand for the dishwasher; (c) comparison between the estimated and ground truth power demand for the fridge; (d) comparison between the estimated and ground truth power demand for the kettle; (e) comparison between the estimated and ground truth power demand for the microwave; (f) comparison between the estimated and ground truth power demand for the washing machine. 

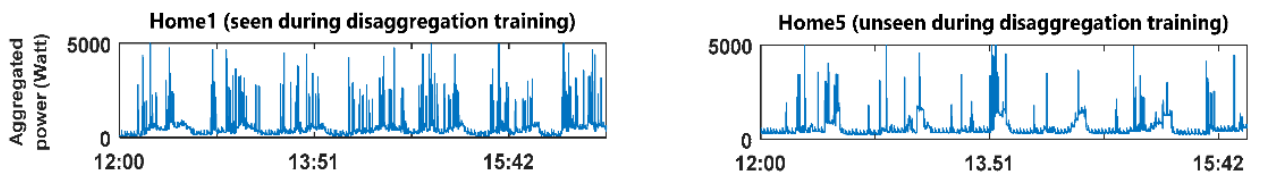

(a)

Estimated
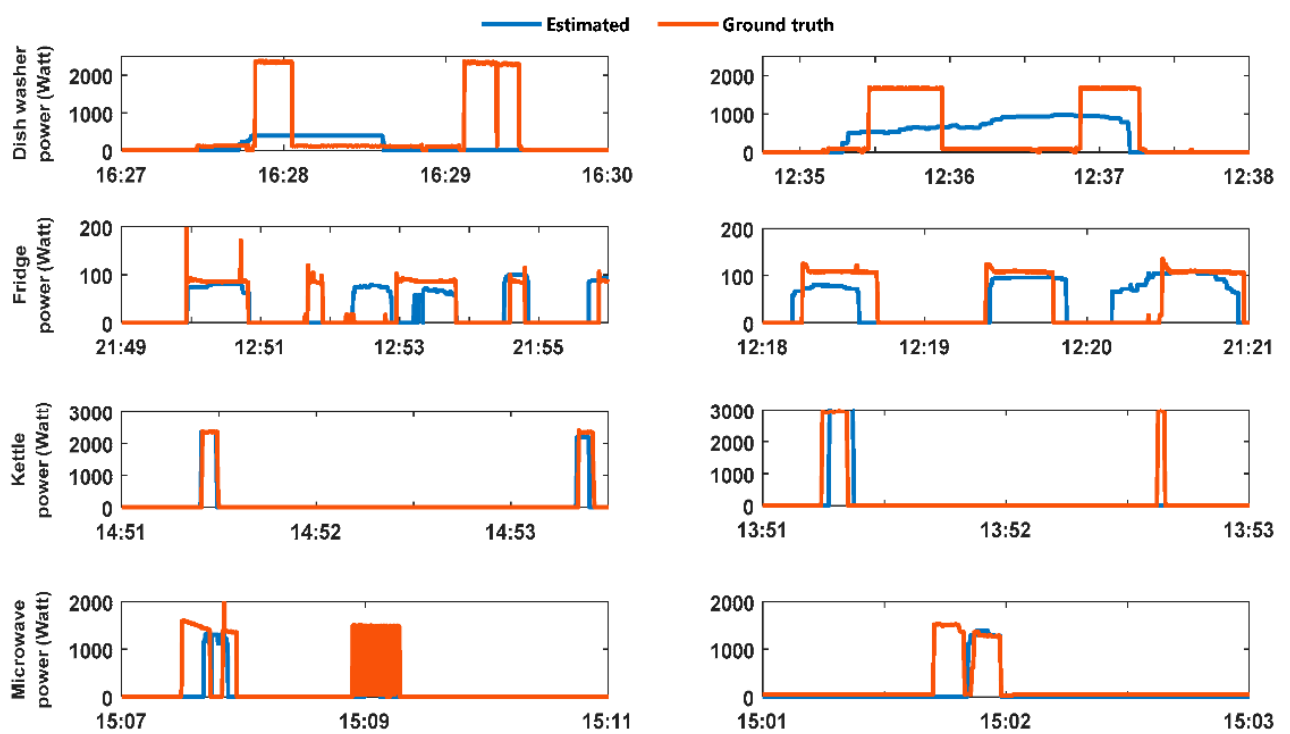

(e)
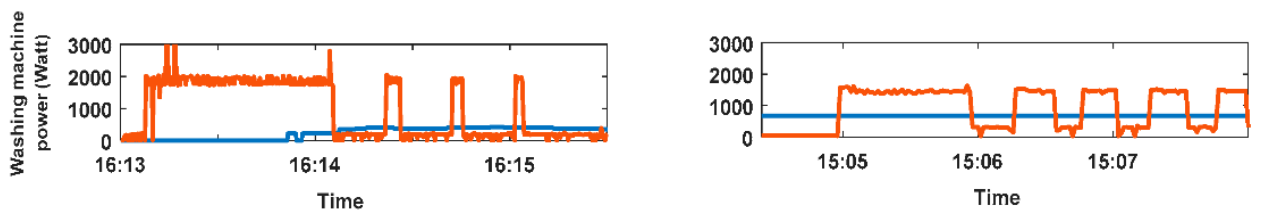

Figure 5. Shows the energy disaggregation algorithm output by using RECTANGLES technique. (a) Aggregated power consumption for the home; (b) wish washer estimated and Ground truth power demand; (c) fridge estimated and Ground truth power demand; (d) kettle estimated and Ground truth power demand; (e) microwave estimated and Ground truth power demand; (f) Washing machine estimated and Ground truth power demand.

\subsubsection{Recurrent Neural Network (RNN or LSTM)}

A recurrent neural network (RNN) is a type of artificial neural network where relations between units form a directed graph along a sequence. This allows it to exhibit dynamic temporal behavior in a time sequence. Different from feedforward neural networks, RNNs can use their internal memory to process sequences of inputs.

Figure 6 shows the output result of the LSTM energy disaggregation algorithm. The figure is divided into two columns. The LHS column has the analysis for the home whose data was available during the training of the disaggregation algorithm. The right-hand side (RHS column have the analysis for the home whose data was not available during the training of the disaggregation algorithm. The figure has six rows described as follows: (a) Aggregated power consumption for the home; (b) comparison between the estimated and ground truth power demand for the dishwasher; (c) comparison between the estimated and ground truth power demand for the fridge; (d) comparison between the estimated and ground truth power demand for the kettle; (e) comparison between the estimated and ground truth power demand for the microwave; (f) comparison between the estimated and ground truth power demand for the washing machine. Table 1 , summarize the compression for the disaggregating algorithms and its performance to for the seen and unseen data provided in Figures 2-6. 

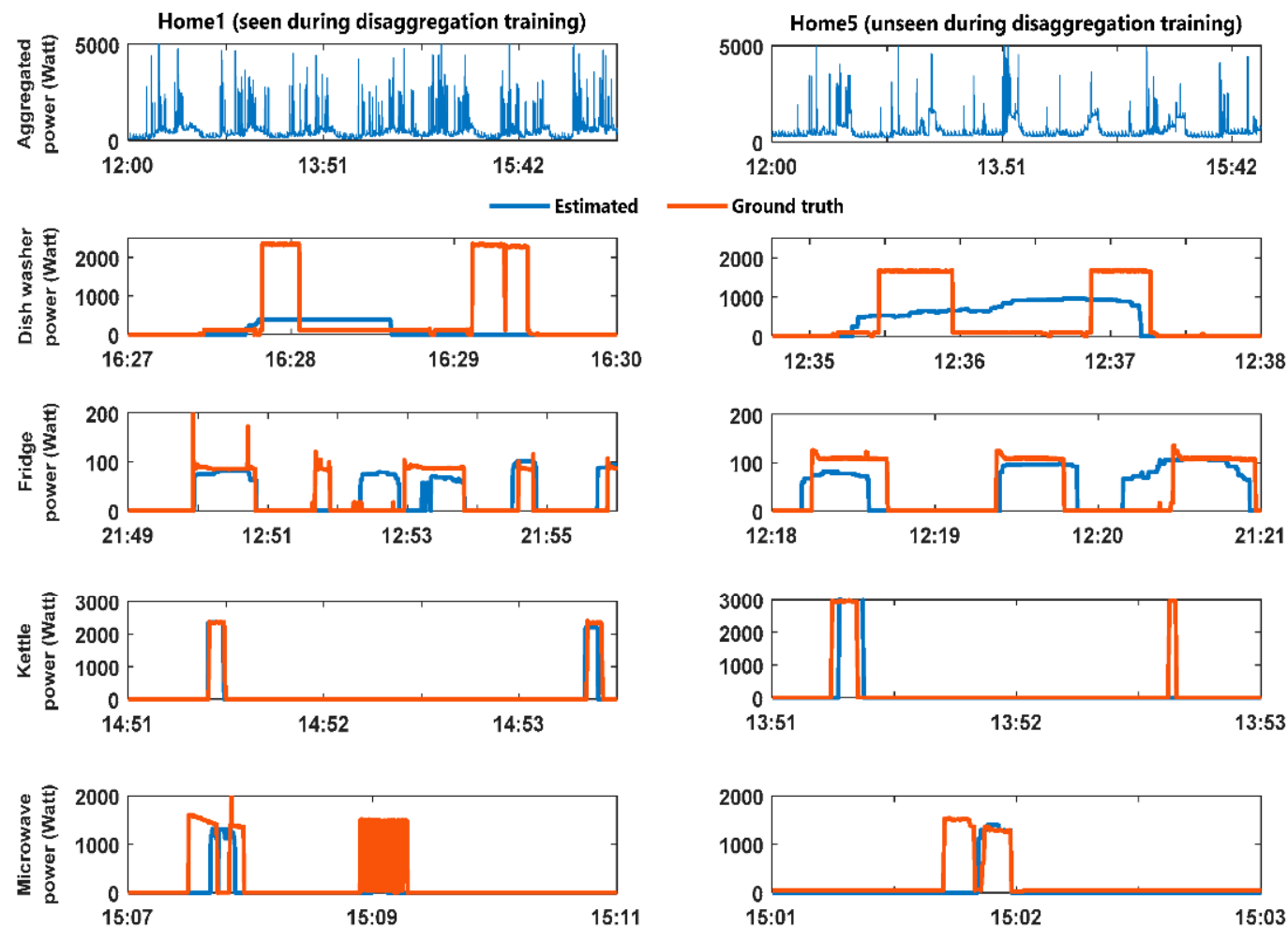

(e)
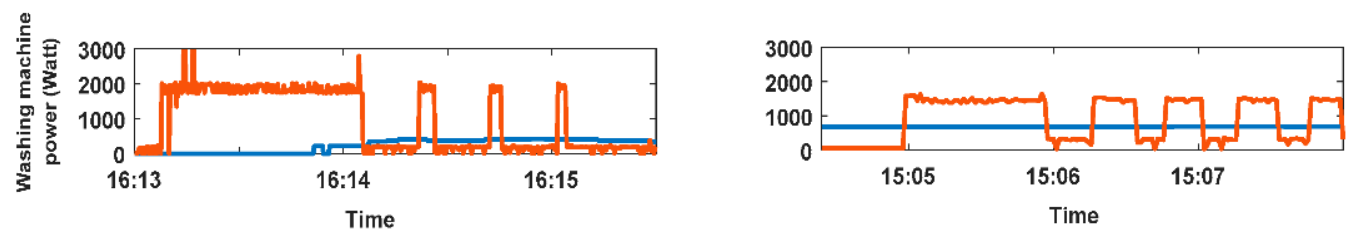

Figure 6. Shows the energy disaggregation algorithm output by using recurrent neural network (RNN) or LSTM technique. (a) Aggregated power consumption for the home; (b) dishwasher estimated and Ground truth power demand; (c) fridge estimated and Ground truth power demand; (d) kettle estimated and Ground truth power demand; (e) microwave estimated and Ground truth power demand; (f) washing machine estimated and Ground truth power demand.

Table 1. Comparison between the disaggregation techniques.

\begin{tabular}{lll}
\hline \multicolumn{1}{c}{ Method } & \multicolumn{1}{c}{ Pros } & \multicolumn{1}{c}{ Cons } \\
\hline Autoencoder & $\begin{array}{l}\text { Succeeded with fridge and kettle } \\
\text { for seen and unseen cases }\end{array}$ & $\begin{array}{l}\text { Fails with dishwasher, microwave and } \\
\text { washing machine for seen and unseen cases }\end{array}$ \\
\hline LSTM & $\begin{array}{l}\text { Succeeded with fridge and kettle } \\
\text { for seen and unseen cases }\end{array}$ & $\begin{array}{l}\text { Fails with dishwasher, microwave and } \\
\text { washing machine for seen and unseen cases }\end{array}$ \\
\hline Rectangles & $\begin{array}{l}\text { Succeeded with dishwasher, } \\
\text { fridge, and kettle for seen and } \\
\text { unseen cases }\end{array}$ & $\begin{array}{l}\text { Fails with microwave and washing machine } \\
\text { for seen and unseen cases }\end{array}$ \\
\hline CO & $\begin{array}{l}\text { Succeeded with fridge only for } \\
\text { seen and unseen cases }\end{array}$ & $\begin{array}{l}\text { Fails with all other appliances in seen and } \\
\text { unseen case. }\end{array}$ \\
\hline FHMM & $\begin{array}{l}\text { Succeeded with fridge for the seen } \\
\text { case only }\end{array}$ & $\begin{array}{l}\text { Fails with all other appliances in both seen } \\
\text { and unseen cases }\end{array}$ \\
\hline
\end{tabular}




\subsection{Disaggregation Stage Analysis}

To identify the best disaggregation algorithm was used in the ED stage. Seven common classification metrics represented through Equations (7)-(23). These seven different accuracy measures are the well-known metrics for evaluating the energy disaggregation techniques [36,37].

$$
\begin{aligned}
& T P=\text { number of true positive } \\
& F P=\text { number of false positive } \\
& F P=\text { number of false negative }
\end{aligned}
$$

$P=$ number of positive in the ground truth

$N=$ number of negative in the ground truth

$$
\begin{gathered}
\text { recall }=\frac{T P}{T P+F N} \\
\text { precision }=\frac{T P}{T P+F P}
\end{gathered}
$$

$\mathrm{F} 1=2 \times \frac{\text { prcision } \times \text { recall }}{\text { precision }+ \text { recall }}$

$$
\text { accuracy }=\frac{T P+T N}{P+N}
$$

$E=$ total actual energy

$\hat{E}=$ total actual energy

$y_{t}^{(i)}=$ appliance $i$ actual power at time $t$

$\hat{y}_{t}^{(i)}=$ appliance $i$ estimated power at timet

$\bar{y}_{t}=$ aggregated actual power at time $t$

relative error in total energy $=\frac{|\hat{E}-E|}{\max (E, \hat{E})}$

$$
\text { mean absolute error }=\frac{1}{T} \sum_{t=1}^{T}\left|\hat{y}_{t}-y_{t}\right|
$$

the proportion of total energy correctly assigned $=1-\frac{\sum_{t=1}^{T} \sum_{i=1}^{n}\left|\hat{y}_{t}^{(i)}-y_{t}^{(i)}\right|}{2 \sum_{t=1}^{T} \bar{y}_{t}}$

Figure 7 shows the comparison between the disaggregation analyses for the home which was seen during training. Figure 8 shows the comparison between the disaggregation analyses for the home which was unseen during training. The denoising autoencoder and RECTANGLES outperform LSTM, FHMM, and CO in most of the metrics throughout the five appliances. Figures 7 and 8 are divided into five columns and seven rows. The five columns represent five appliances labeled from the left as follows: Dishwasher; fridge; kettle; microwave; washing machine. The seven rows labeled from upper to lower as follows: F1 score Equation (14); precision score Equation (13); recall score Equation (12); accuracy score Equation (15); relative error in total energy Equation (21); proportion of total energy correctly assigned Equation (23); mean absolute error Equation (22). Therefore, the result at position $(1,1)$ represents the F1 score for the dishwasher with five different data mining energy disaggregation algorithms. These five algorithms were the legend at the footer of the figures. 


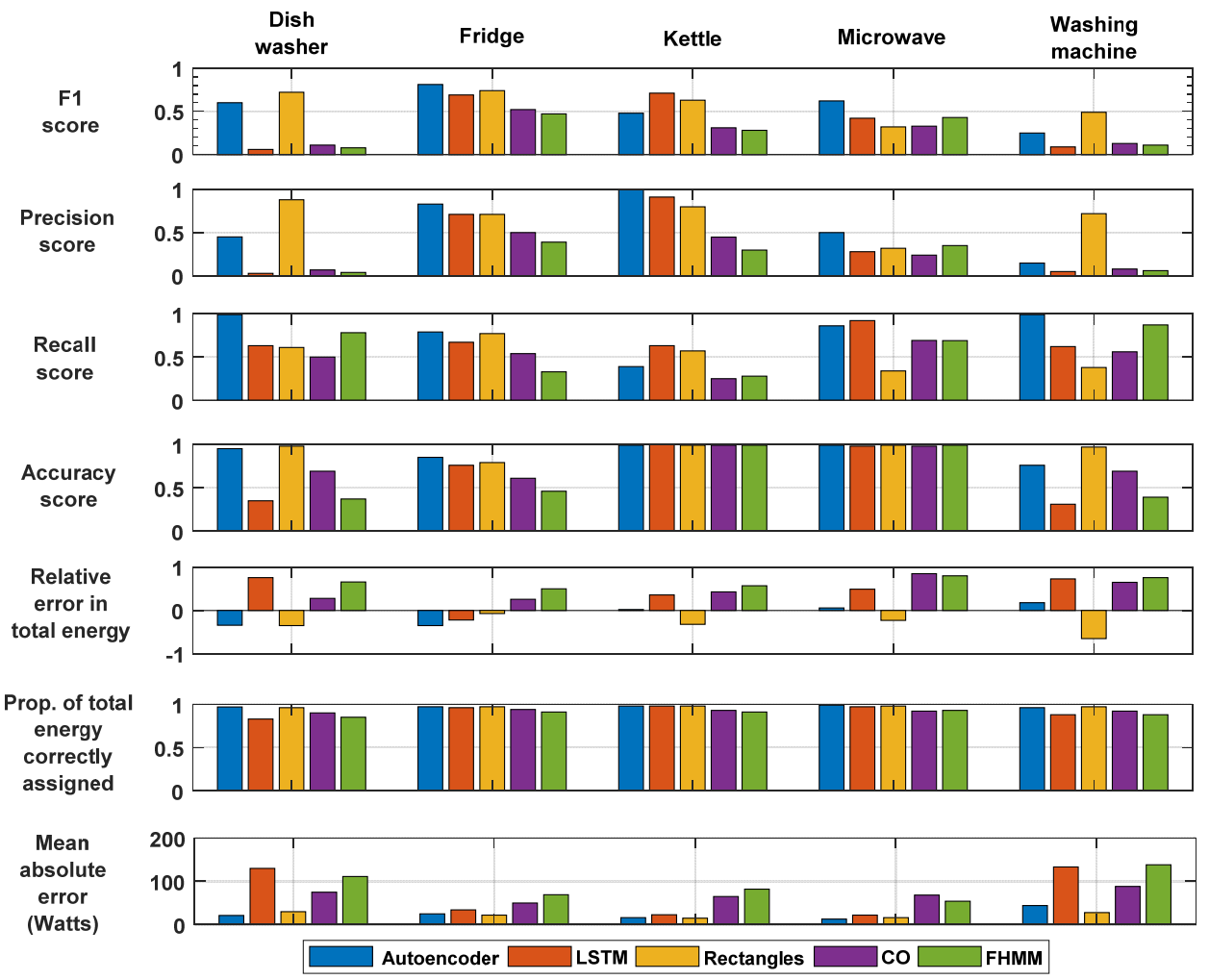

Figure 7. Energy disaggregation performance analysis for home seen during training.

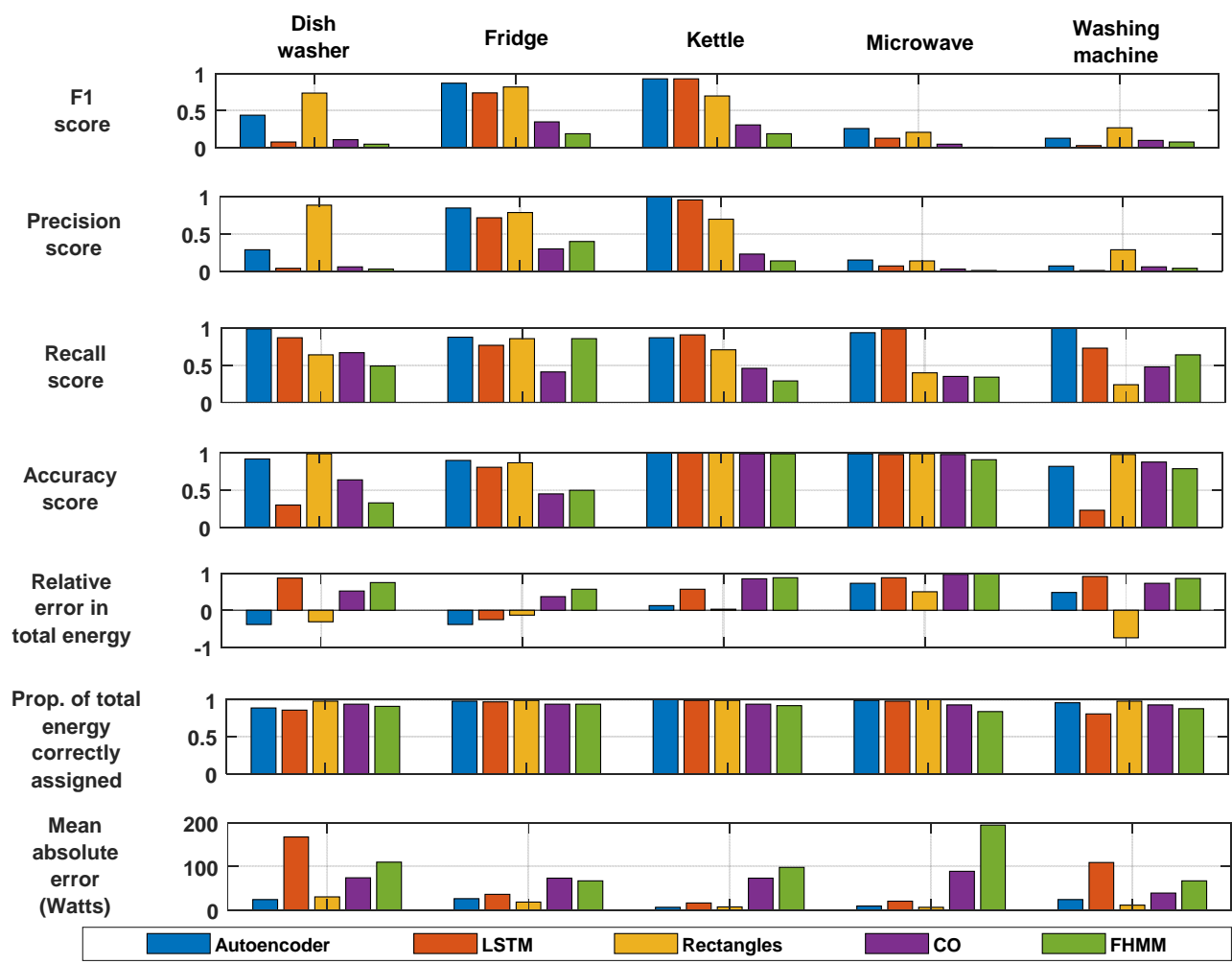

Figure 8. Energy disaggregation performance analysis for home unseen during training. 


\section{The Implemented Short-Term Load Forecasting}

A feed-forward Neural Network using the Levenberg-Marquardt backpropagation algorithm was employed. The Neural network consists of one input layer, three hidden layers, and one node at the output layer to predicted the aggregated power demand for an hour ahead. The input layer has eleven inputs which match the data utilized for load prediction. These input data consists of (five inputs from the disaggregation stage and six inputs from the aggregated demand of the home for the current and previous hours of historical data. The five inputs represent the load demand of the five major appliances in the home at the present hour which extracted from the energy disaggregation stage (dishwasher, fridge, kettle, microwave, and washing machine). The six inputs represent the current and historical consumption hours. Three inputs include the current hour power demand, one for an hour before, one for two hours earlier, and another three inputs include one for a day earlier, one for the $23 \mathrm{~h}$ earlier, and one for $22 \mathrm{~h}$ earlier. The reason for selecting six inputs from the aggregated data is that we picked two groups of three inputs. One group will cover the most recent three hours, and the other group covers the early three hours in the last $24 \mathrm{~h}$. Each three inputs will cover three hours to cover double of the maximum interval of the time cycle of the washing machine which has the tallest time interval reach to $90 \mathrm{~min}$ [25]. Regarding the data structure, there are significant changes in the inputs and output ranges. Thus, all the input and output data have been normalized to avoid saturation of the FFANN. Normalization is done using Equation (24).

$$
P_{n}=P / P_{\max }
$$

where $p_{n}$ is the normalized power value, $p$ is the actual power value, $P_{\max }$ is the peak power. For training, validation, and testing of the neural network, the data divided to $70 \%$ for training, $15 \%$ for validating, and $15 \%$ for testing.

In many ways, this test network presents a challenging forecasting case, and these are all drawn from the real UK dataset.

\section{Simulation Results}

Figures 9 and 10 demonstrate the actual load and the forecasted load by different methods for the home seen during the ED training and unseen during ED training, respectively. In order to assess the performance of the proposed method in conducting STLF for residential households, three widely used metrics were employed, including root mean squared error (RMSE), normalized root mean squared error, and mean absolute error. The three performance metrics are introduced in Equations (25)-(27). These three metrics describe the performance of the forecaster from different view [38,39]. The RMES is good for getting average error considering the error direction. In another word, the RMSE can give the idea about the average error between the predicted and actual signal regardless of the direction of the error. Additionally, the NRMSE gives the same description, but by normalized values which allows the comparison between different systems (two home with two different power rating). However, the MAE gives the average error over a period concerning the direction which could give a good idea of the accumulated error in the forecasted energy.

$$
\begin{gathered}
R M S E=\sqrt{\frac{\sum_{t=1}^{N}\left(\hat{y}_{t}-y_{t}\right)^{2}}{N}} \\
N R M S E=\frac{R M S E}{y_{\max }-y_{\min }} \\
M A E=\frac{\sum_{t=1}^{T}\left|\hat{y}_{t}-y_{t}\right|}{N}
\end{gathered}
$$




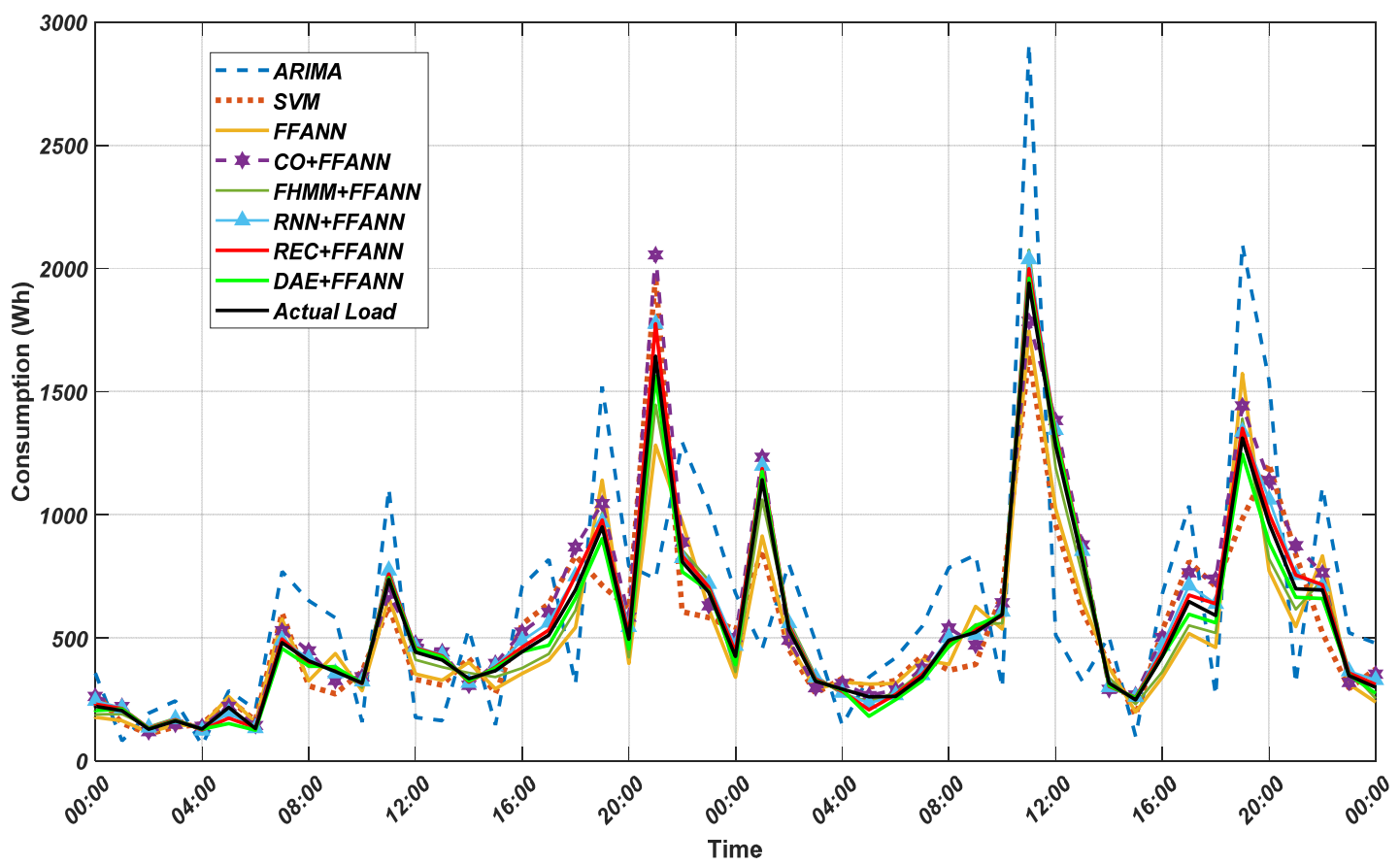

Figure 9. The actual and forecasted load by different methods for the home seen during the ED training.

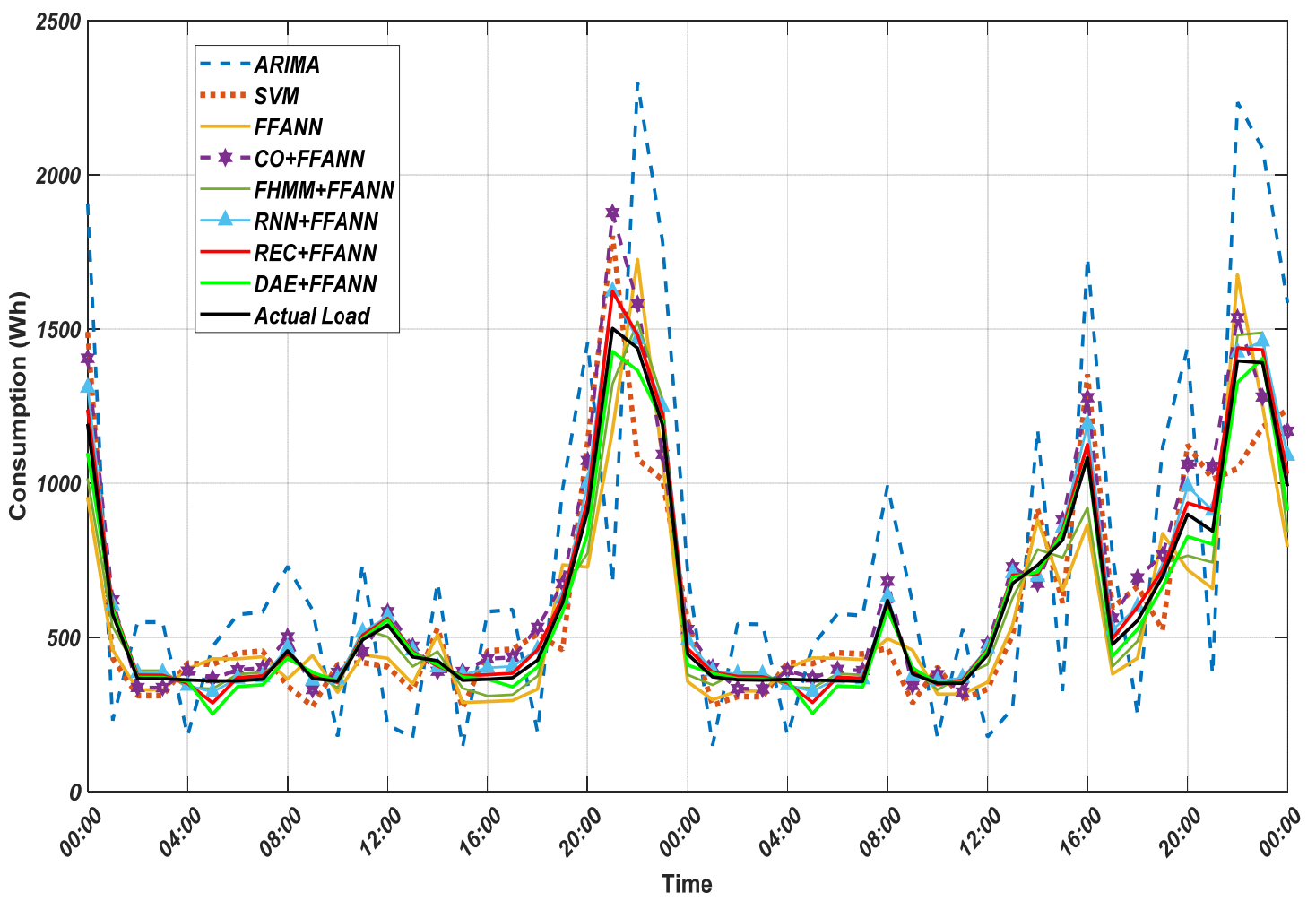

Figure 10. The actual and forecasted load by a different method for the home was unseen during the ED training.

Tables 2 and 3 compare the performance of the proposed approach regarding RMSE, NRMSE, and MAE with the current state of the art techniques, i.e., AIRMA, SVM, and FFANN. As illustrated, the five proposed approaches; DAE + FFANN, REC + FFANN, RNN + FFANN, FHMM + FFANN, 
and CO + FFANN outperform FFANN, SVM, and ARIMA in all metrics used. In case of Table 2 which should be the worst because the data was unseen during the energy disaggregation stage. The proposed (REC + FFANN) brings 91.13\% reduction in RMSE and NRMSE, 92.36\% reduction of MAE as compared with ARIMA.

Table 2. Load Forecasting Performance Comparison for the seen home.

\begin{tabular}{cccc}
\hline Architecture & RMSE (kwh) & NRMSE & MAE (kwh) \\
\hline ARIMA & 0.3831 & 0.1906 & 0.2935 \\
SVM & 0.1369 & 0.0749 & 0.1145 \\
FFANN & 0.1145 & 0.0627 & 0.0942 \\
CO + FFANN & 0.0877 & 0.0480 & 0.0641 \\
FHMM + FFANN & 0.0580 & 0.0318 & 0.0457 \\
RNN + FFANN & 0.0382 & 0.0209 & 0.2880 \\
DAE + FFANN & 0.0309 & 0.0169 & 0.0228 \\
REC + FFANN & 0.0291 & 0.0159 & 0.0221 \\
\hline
\end{tabular}

Table 3. Load forecasting performance comparison for the unseen home.

\begin{tabular}{cccc}
\hline Architecture & RMSE (kwh) & NRMSE & MAE (kwh) \\
\hline ARIMA & 0.4280 & 0.1854 & 0.3506 \\
SVM & 0.1665 & 0.0722 & 0.1359 \\
FFANN & 0.1397 & 0.0605 & 0.1112 \\
CO + FFANN & 0.1145 & 0.0496 & 0.0770 \\
FHMM + FFANN & 0.0769 & 0.0333 & 0.0558 \\
RNN + FFANN & 0.0507 & 0.0219 & 0.0354 \\
DAE + FFANN & 0.0432 & 0.0187 & 0.0287 \\
REC + FFANN & 0.0372 & 0.0161 & 0.0268 \\
\hline
\end{tabular}

\section{Conclusions}

In this paper, an improved load demand forecasting technique utilizing a preprocessing stage of energy disaggregation techniques combined with FFANN are proposed. This proposed approach implemented for household's STLF under high uncertainty and volatility associated with customer behavior which is difficult to predict. Five different energy disaggregation techniques; DAE, RECTANGLES, RNN, FHMM, and CO were implemented and evaluated for a data of two different homes. Seven performance metrics were utilized to benchmark the implemented energy disaggregation techniques to give a comprehensive comparison of the performance of the techniques being assessed. The proposed STLF approaches; RECTANGLES + FFANN, DAE + FFANN, RNN + FFANN, FHMM + FFANN, and CO + FFANN outperform FFANN, SVM, and ARIMA in all three benchmark metrics have usually been used in literature to evaluate the performance of STLF. The best approach used for energy disaggregation is denoising autoencoder which directly affected the performance of the STLF at residential household level. A great comparison and performance analysis show that the proposed technique (DEA + FFANN) brings 91.13\% reduction in RMSE and NRMSE, 92.36\% reduction of MAE as compared to ARIMA.

Author Contributions: A.F.E. developed the pre-processing technique for the energy demand forecasting, built the simulation model and performed data analysis. O.A.M. is the main supervisor who leads the project, identifies the ideas, checks the results and edits the manuscript.

Acknowledgments: This work was supported in part by the U.S. Department of Energy and the Office of Naval Research.

Conflicts of Interest: The authors declare no conflict of interest. 


\section{References}

1. Yunus, K.; Thiringer, T.; Chen, P. ARIMA-Based Frequency-Decomposed Modeling of Wind Speed Time Series. IEEE Trans. Power Syst. 2016, 31, 2546-2556. [CrossRef]

2. Jiang, H.; Zhang, Y.; Muljadi, E.; Zhang, J.; Gao, W. A short-term and high-resolution distribution system load forecasting approach using support vector regression with hybrid parameters optimization. IEEE Trans. Smart Grid 2016. [CrossRef]

3. Abu-Elanien, A.E.B.; Salama, M.M.A. A Wavelet-ANN Technique for Locating Switched Capacitors in Distribution Systems. IEEE Trans. Power Deliv. 2009, 24, 400-409. [CrossRef]

4. Korolko, N.; Sahinoglu, Z.; Nikovski, D. Modeling and Forecasting Self-Similar Power Load Due to EV Fast Chargers. IEEE Trans. Smart Grid 2016, 7, 1620-1629. [CrossRef]

5. Zhang, P.; Zhou, X.; Pelliccione, P.; Leung, H. RBF-MLMR: A Multi-Label Metamorphic Relation Prediction Approach Using RBF Neural Network. IEEE Access 2017, 5, 21791-21805. [CrossRef]

6. Zhang, Y.; Zhou, Q.; Sun, C.; Lei, S.; Liu, Y.; Song, Y. RBF Neural Network and ANFIS-Based Short-Term Load Forecasting Approach in Real-Time Price Environment. IEEE Trans. Power Syst. 2008, 23, 853-858. [CrossRef]

7. Kong, W.; Dong, Z.Y.; Hill, D.J.; Luo, F.; Xu, Y. Short-Term Residential Load Forecasting Based on Resident Behaviour Learning. IEEE Trans. Power Syst. 2018, 33, 1087-1088. [CrossRef]

8. Hippert, H.S.; Pedreira, C.E.; Souza, R.C. Neural networks for short-term load forecasting: A review and evaluation. IEEE Trans. Power Syst. 2001, 16, 44-55. [CrossRef]

9. Ahmad, A.S.; Hassan, M.Y.; Abdullah, M.P.; Rahman, H.A.; Hussin, F.; Abdullah, H.; Saidur, R. A review on applications of ANN and SVM for building electrical energy consumption forecasting. Renew. Sustain. Energy Rev. 2014, 33, 102-109. [CrossRef]

10. Teeraratkul, T.; O’Neill, D.; Lall, S. Shape-Based Approach to Household Electric Load Curve Clustering and Prediction. IEEE Trans. Smart Grid 2017. [CrossRef]

11. Ahmad, A.; Javaid, N.; Guizani, M.; Alrajeh, N.; Khan, Z.A. An Accurate and Fast Converging Short-Term Load Forecasting Model for Industrial Applications in a Smart Grid. IEEE Trans. Ind. Inform. 2017, 13, 2587-2596. [CrossRef]

12. Ouammi, A. Optimal Power Scheduling for a Cooperative Network of Smart Residential Buildings. IEEE Trans. Sustain. Energy 2016, 7, 1317-1326. [CrossRef]

13. Ahmed, N.; Levorato, M.; Li, G.-P. Residential Consumer-Centric Demand Side Management. IEEE Trans. Smart Grid 2017. [CrossRef]

14. Ryu, M.S.; Noh, M.J.; Kim, H. Deep Neural Network Based Demand Side Short-Term Load Forecasting. In Proceedings of the 2016 IEEE International Conference on Smart Grid Communications (SmartGridComm), Sydney, NSW, Australia, 6-9 November 2016; p. 6.

15. Tian, P.; Xiao, X.; Wang, K.; Ding, R. A Hierarchical Energy Management System Based on Hierarchical Optimization for Microgrid Community Economic Operation. IEEE Trans. Smart Grid 2016, 7, 2230-2241. [CrossRef]

16. Marino, D.L.; Amarasinghe, K.; Manic, M. Building energy load forecasting using Deep Neural Networks. In Proceedings of the IECON 2016-42nd Annual Conference of the IEEE Industrial Electronics Society, Florence, Italy, 24-27 October 2016; pp. 7046-7051.

17. Shi, H.; Xu, M.; Li, R. Deep Learning for Household Load Forecasting-A Novel Pooling Deep RNN. IEEE Trans. Smart Grid 2017. [CrossRef]

18. Youssef, T.A.; Hariri, M.E.; Elsayed, A.T.; Mohammed, O.A. A DDS-Based Energy Management Framework for Small Microgrid Operation and Control. IEEE Trans. Ind. Inform. 2018, 14, 958-968. [CrossRef]

19. Elsied, M.; Oukaour, A.; Youssef, T.; Gualous, H.; Mohammed, O. An advanced real-time energy management system for microgrids. Energy 2016, 114, 742-752. [CrossRef]

20. Hart, G.W. Nonintrusive appliance load monitoring. Proc. IEEE 1992, 80, 1870-1891. [CrossRef]

21. Hart, G.W. Residential energy monitoring and computerized surveillance via utility power flows. IEEE Technol. Soc. Mag. 1989, 8, 12-16. [CrossRef]

22. Roos, J.G.; Lane, I.E.; Botha, E.C.; Hancke, G.P. Using neural networks for non-intrusive monitoring of industrial electrical loads. In Proceedings of the 1994 IEEE Instrumentation and Measurement 
Technolgy Conference (Cat. No.94CH3424-9), 10th Anniversary IMTC/94 Advanced Technologies in I \& M, Hamamatsu, Japan, 10-12 May 1994; Volume 3, pp. 1115-1118.

23. Yang, H.T.; Chang, H.H.; Lin, C.L. Design a Neural Network for Features Selection in Non-intrusive Monitoring of Industrial Electrical Loads. In Proceedings of the 2007 11th International Conference on Computer Supported Cooperative Work in Design, Melbourne, VIC, Australia, 26-28 April 2007; pp. 1022-1027.

24. Lin, Y.H.; Tsai, M.S. A novel feature extraction method for the development of nonintrusive load monitoring system based on BP-ANN. In Proceedings of the 2010 International Symposium on Computer, Communication, Control and Automation (3CA), Tainan, Taiwan, 5-7 May 2010; Volume 2, pp. 215-218.

25. Kelly, J.; Knottenbelt, W. The UK-DALE dataset, domestic appliance-level electricity demand and whole-house demand from five UK homes. Sci. Data 2015, 2, 150007. [CrossRef] [PubMed]

26. Batra, N.; Kelly, J.; Parson, O.; Dutta, H.; Knottenbelt, W.; Rogers, A.; Singh, A.; Srivastava, M. NILMTK: An open source toolkit for non-intrusive load monitoring. In Proceedings of the Fifth International Conference on Future Energy Systems (ACM e-Energy), Cambridge, UK, 11-13 June 2014; pp. 265-276.

27. Parson, O. Unsupervised Training Methods for Non-Intrusive Appliance Load Monitoring from Smart Meter Data. Doctoral Thesis, University of Southampton, Southampton, UK, 2014.

28. Chen, M.; Liew, S.C.; Shao, Z.; Kai, C. Markov Approximation for Combinatorial Network Optimization. IEEE Trans. Inf. Theory 2013, 59, 6301-6327. [CrossRef]

29. Rouvellou, I.; Hart, G.W. Topology identification for traffic and configuration management in dynamic networks. In Proceedings of the Eleventh Annual Joint Conference of the IEEE Computer and Communications Societies, IEEE INFOCOM '92, Florence Italy, 4-8 May 1992; Volume 3, pp. 2197-2204.

30. Lin, Y.H.; Tsai, M.S. Non-Intrusive Load Monitoring by Novel Neuro-Fuzzy Classification Considering Uncertainties. IEEE Trans. Smart Grid 2014, 5, 2376-2384. [CrossRef]

31. Bonfigli, R.; Principi, E.; Squartini, S.; Fagiani, M.; Severini, M.; Piazza, F. User-aided footprint extraction for appliance modelling in Non-Intrusive Load Monitoring. In Proceedings of the 2016 IEEE Symposium Series on Computational Intelligence (SSCI), Athens, Greece, 6-9 December 2016; pp. 1-8.

32. Kong, W.; Dong, Z.Y.; Hill, D.J.; Luo, F.; Xu, Y. Improving Nonintrusive Load Monitoring Efficiency via a Hybrid Programing Method. IEEE Trans. Ind. Inform. 2016, 12, 2148-2157. [CrossRef]

33. Makonin, S.; Popowich, F.; Bajić, I.V.; Gill, B.; Bartram, L. Exploiting HMM Sparsity to Perform Online Real-Time Nonintrusive Load Monitoring. IEEE Trans. Smart Grid 2016, 7, 2575-2585. [CrossRef]

34. Egarter, D.; Bhuvana, V.P.; Elmenreich, W. PALDi: Online Load Disaggregation via Particle Filtering. IEEE Trans. Instrum. Meas. 2015, 64, 467-477. [CrossRef]

35. Mauch, L.; Yang, B. A novel DNN-HMM-based approach for extracting single loads from aggregate power signals. In Proceedings of the 2016 IEEE International Conference on Acoustics, Speech and Signal Processing (ICASSP), Shanghai, China, 20-25 March 2016; pp. 2384-2388.

36. Pereira, L.; Nunes, N.J. A Comparison of Performance Metrics for Event Classification in Non-Intrusive Load Monitoring. In Proceedings of the 2017 IE International Conference on Smart Grid Communications, Dresden, Germany, 23-27 October 2017.

37. Zhang, L.; Liu, Y.; Chen, G.; He, X.; Guo, X. Assessment Metrics for Unsupervised Non-intrusive Load Disaggregation Learning Algorithms. In Practical Applications of Intelligent Systems; Wen, Z., Li, T., Eds.; Springer: Berlin/Heidelberg, Germany, 2014; Volume 279, pp. 197-206.

38. Ceperic, E.; Ceperic, V.; Baric, A. A Strategy for Short-Term Load Forecasting by Support Vector Regression Machines. IEEE Trans. Power Syst. 2013, 28, 4356-4364. [CrossRef]

39. Li, S.; Wang, P.; Goel, L. A Novel Wavelet-Based Ensemble Method for Short-Term Load Forecasting with Hybrid Neural Networks and Feature Selection. IEEE Trans. Power Syst. 2016, 31, 1788-1798. [CrossRef]

(C) 2018 by the authors. Licensee MDPI, Basel, Switzerland. This article is an open access article distributed under the terms and conditions of the Creative Commons Attribution (CC BY) license (http:// creativecommons.org/licenses/by/4.0/). 